\title{
Hipertensión arterial en la infancia. Recomendaciones para su diagnóstico y tratamiento. Parte 2 Rama de Nefrología Infantil, Sociedad Chilena de Pediatría
}

\author{
Blood hypertension in children. Guideliness for diagnosis and treatment. Part 2 \\ Pediatric Nephrology Branch, Chilean Pediatric Society
}

\author{
Paulina Salas $^{\mathrm{a}}$, Claudia González ${ }^{\mathrm{b}, \mathrm{i}}$, Daniela Carrillo ${ }^{\mathrm{b}, \mathrm{e}}$, Lillian Bolte ${ }^{\mathrm{c}}$, Marlene Aglony ${ }^{\mathrm{d}}$, Soledad Peredo ${ }^{\mathrm{e}}$, \\ Ximena Ibarra ${ }^{\mathrm{e}}$, Angélica Rojo ${ }^{\mathrm{a}}$, Angela Delucchi ${ }^{\mathrm{f}}$, Viola Pinto ${ }^{\mathrm{a}, \mathrm{g}}$, Carlos Saieh$^{\mathrm{g}}$, María L. Ceballos ${ }^{\mathrm{f}}$
}

\author{
aHospital Exequiel González Cortés \\ bHospital Sótero del Río \\ cHospital Roberto del Río \\ ¿Sociedad Chilena de Hipertensión \\ eHospital Clínico Pontificia Universidad Católica de Chile \\ ${ }^{\mathrm{f}}$ Hospital Luis Calvo Mackenna \\ 'Clínica Las Condes \\ i Pontificia Universidad Católica de Chile
}

\section{Resumen}

La hipertensión arterial (HTA) en niños y adolescentes es una importante patología, de reservado pronóstico, asociada a factores modificables y no modificables. La prevalencia estimada es de aproximadamente un 3,5\%, la cual va aumentando progresivamente con la edad. El método ideal para su diagnóstico es la medición de la presión arterial (PA) con instrumentos auscultatorios. De acuerdo a la Academia Americana de Pediatría (AAP) la PA debe ser medida en niños mayores de 3 años una vez al año, y en niños menores de 3 años, si presentan factores de riesgo. Una vez confirmada la HTA, la evaluación debe dirigirse hacia la detección de una enfermedad causal y/o a la búsqueda de factores de riesgo asociados a una HTA primaria. El objetivo del tratamiento de la HTA primaria y secundaria en pediatría es lograr un nivel de PA que disminuya el riesgo de daño de los órganos blanco. Las opciones terapéuticas incluyen: tratamiento según etiología específica, no farmacológico y farmacológico. En esta Guia se presenta la posición de la Rama de Nefrología de la Sociedad Chilena de Pediatría con el objetivo de orientar a pediatras y nefrólogos infantiles en correcto manejo de la HTA en la infancia. En esta segunda parte se presentan las recomendaciones sobre el tratamiento antihipertensivo, haciendo énfasis en los cambios de estilo de vida.
Palabras clave:

Hipertensión arterial; riesgo cardiovascular; órgano blanco; antihipertensivos; DASH

Correspondencia:

Paulina Salas del Campo

psalasdelc@gmail.com 


\begin{abstract}
Hypertension (HTN) in children and adolescents is an important pathology, of, guarded prognosis, associated with modifiable and non-modifiable factors. The estimated prevalence is around 3.5\% which increases progressively with age. The ideal method for its diagnosis is the measurement of blood pressure (BP) with auscultatory instruments. According to the American Academy of Pediatrics (AAP), BP should be measured in children older than three years of age once a year, and in children younger than three years of age if they present risk factors. Once the HTN is confirmed, the evaluation should be directed towards the detection of a causative disease and/or the search for risk factors associated with a primary HTN. The objective of treating primary and secondary HTN in pediatrics is to achieve a BP level that decreases the risk of target organ damage. Therapeutic options include treatment according to specific etiology, non-pharmacological and pharmacological one. This paper presents the position of the Chilean Society of Pediatrics Nephrology Branch with the aim of guiding pediatricians and pediatric nephrologists in the correct management of HTN in childhood. In this second part, recommendations on antihypertensive treatment are presented with an emphasis on lifestyle changes.
\end{abstract}

\section{Keywords:} hypertension; cardiovascular risk; target organ; antihypertensives; DASH

\section{Abreviaturas}

HTA: hipertensión arterial.

PA: presión arterial.

Pc: percentil.

HVI: hipertrofia ventricular izquierda.

ERC: enfermedad renal crónica.

IECA: inhibidores de la enzima convertidora.

ARAII: antagonista de los receptores de angiotensina II.

DM: diabetes mellitus.

TG: triglicéridos.

LDL: lipoproteínas de baja densidad.

SRAA: sistema renina angiotensina aldosterona.

RVP: resistencia vascular periférica.

\section{Tratamiento de Hipertensión Arterial}

El objetivo del tratamiento de la Hipertensión Arterial (HTA) primaria y secundaria en pediatría es lograr un nivel de Presión Arterial (PA) que disminuya el riesgo de daño de órgano blanco. Es así como, las guías actuales recomiendan una disminución de PA bajo percentil (pc) 90 y en adolescentes bajo 130/80, para lograr este objetivo, además de disminuir riesgo cardiovascular e HTA en edad adulta ${ }^{1}$.

Las opciones terapéuticas incluyen: tratamiento según etiología específica, no farmacológico y farmacológico.

\section{VI a. Tratamiento no farmacológico} ("cambios de estilo de vida")

Tanto en adultos como en niños, existen evidencias que soportan estas medidas como el pilar del tratamiento de la HTA. Este tratamiento incluye ${ }^{1,2}$ :
Actividad física (AF): se sugiere:

- Limitar actividades sedentarias $<2$ h/día.

- Realizar actividad física moderada o vigorosa 3-5 veces a la semana, 30-60 min por sesión.

- La actividad física debiera ser principalmente aeróbica, pudiendo incorporar ejercicios de resistencia, con un programa de acondicionamiento físico.

- La participación en deportes competitivos sólo se limita en HTA II no controlada.

\section{Alimentación saludable: se sugiere:}

- Disminuir el consumo de sal a 3 g/día con lo cual se logra una significativa caída en la PA de pacientes hipertensos y normotensos, independiente del sexo y la edad.

- Dieta basada en las recomendaciones DASH (Dietary Approaches to Stop Hypertension Study), que incluye altos aportes de frutas, verduras, granos, carnes magras y pescado, asociada a restricción de grasas saturadas y azúcar (tabla 1).

Apoyo psicológico: El objetivo del apoyo de salud mental, es realizar intervenciones motivacionales para que el niño o adolescente pueda lograr cambios en la conducta alimentaria y en la práctica de actividad física que sean duraderas en el tiempo y lograr así adherencia a los hábitos de vida saludables.

Otras intervenciones:

- Evitar fumar.

- Evitar estimulantes del sistema simpático como: cafeína, pseudoefedrina, cocaína, AINES, hormonas, bebidas energéticas.

- Mejorar la calidad del sueño. 
Tabla 1. Recomendaciones de dieta DASH

\begin{tabular}{lc}
\hline Alimento & Porciones diarias \\
\hline Frutas y vegetales & $4-5$ \\
Productos lácteos descremados & $\geq 2$ \\
Granos enteros & 6 \\
Pescado, pollo, carnes rojas desgrasadas & $\leq 2$ \\
Legumbres y frutos secos & 1 \\
Aceites y grasas & $2-3$ \\
Azucares & $\leq 1$ \\
Sal & $<2.300 \mathrm{mg} / \mathrm{día}$ \\
\hline
\end{tabular}

Tabla adaptada de referencia (5).

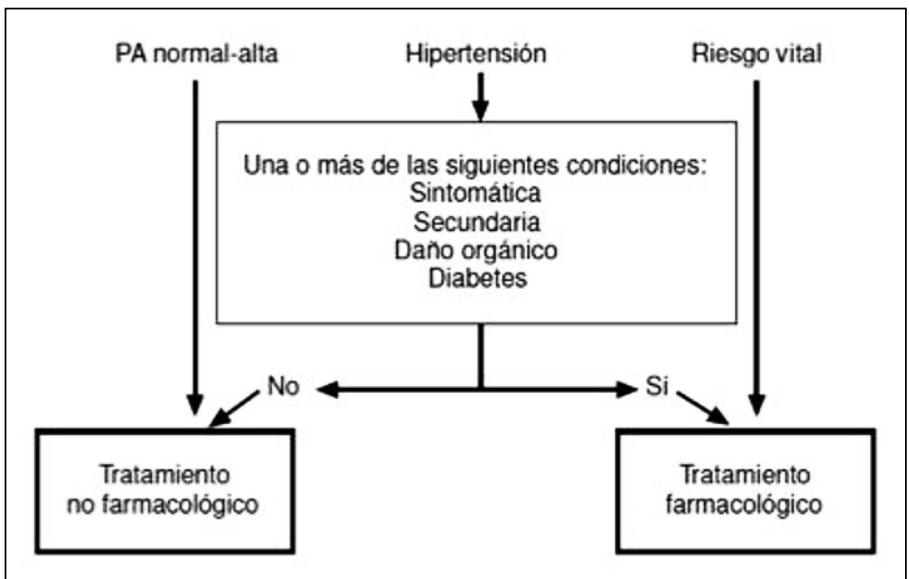

Figura 1. Resumen de tratamiento en HTA.

\section{VI b. Tratamiento farmacológico de Hipertensión Arterial}

El tratamiento farmacológico de la HTA ha demostrado la efectividad de las drogas en disminuir la PA también en la edad pediátrica. Sin embargo, sólo en los últimos años se han publicado estudios que demuestran cuál droga es más efectiva en disminuir riesgo cardiovascular, medido a través de marcadores intermedios como: HVI, microalbuminuria, progresión ERC e índice grosor intima media.

El inicio de terapia farmacológica se extrapola de lo observado en adultos, ya que no hay respaldo de estudios clínicos pediátricos que indiquen el momento de iniciarla. Además, se debe considerar la etiología de la HTA, severidad, sintomatología asociada, daño de órgano blanco y comorbilidades existentes.

En líneas generales, no existen diferencias significativas en la eficacia de cada droga. Sin embargo, cabe destacar que estudios de daño endotelial en adultos demuestran mejoría con el uso de IECA, ARA II, bloqueadores canales de calcio y espironolactona, no así con betabloqueadores.

Las principales indicaciones para el uso de medica- mentos hipotensores son (ver figura 1$)^{3-6}$ :

- HTA primaria, que persiste a pesar de modificaciones en el estilo de vida.

- Daño de órgano blanco.

- HTA sintomática.

- Presencia de comorbilidades que aumentan riesgo cardiovascular: obesidad, ERC, DM.

- HTA secundaria.

\section{Elección del medicamento}

Se sugiere iniciar monoterapia para evitar descenso brusco de PA, se puede ir aumentando en forma gradual la dosis cada 2-4 semanas, hasta llegar a dosis máxima del medicamento o hasta aparición de efectos secundarios. Si bajo esas circunstancias, la PA no logra controlarse, debe agregarse un segundo hipotensor.

El paciente debe ser controlado cada 4-6 semanas hasta lograr estabilización de la PA.

Es importante conocer al menos un hipotensor de cada clase. Los cuales se describen brevemente a continuación:

\section{Betabloquedores}

Hay pocos estudios que demuestran eficacia en comparación a placebo. Se observa reducción de PA con un $5 \%$ de efectos adversos ${ }^{7}$. Su uso no mejora la disfunción endotelial. Se recomienda en casos de HTA por hipertiroidismo y en feocromocitoma después de alfa bloqueo (para evitar actividad alfa adrenérgica sin oposición). Evitar en asma y diabetes mellitus. Precaución en obesidad, ya que se ha asociado con aumento de peso $^{6}$.

Carvedilol ha demostrado reducir la morbimortalidad en pacientes con miocardiopatía dilatada, por lo que se sugiere su uso en pacientes con insuficiencia cardiaca $^{8}$.

\section{Antagonistas canal calcio (ACC)}

Existen estudios con diltiazem, verapamilo, nifedipino, amlodipino, felodipino e isradipina ${ }^{9}$. El más estudiado y avalado actualmente en pediatría es el amlodipino, con reducción significativa de PA vs placebo ${ }^{9,10}$. La farmacocinética cambia en niños mayores de 6 años requiriendo más dosis por kilo que los más pequeños.

\section{Inhibidores de enzima convertidora de angiotensina (IECA)}

Captopril ampliamente estudiado, pero requiere de varias dosis en el día, por lo que se ha reemplazado por medicamentos de efecto más prolongado como enalapril, lisinopril, quinalapril ${ }^{12-14}$.

\section{Antagonistas del receptor de angiotensina II (ARA II)}

Existen trabajos con losartán y valsartán, con buenos resultados en la disminución de PA. Los úl- 
timos estudios utilizan valsartán desde los 6 meses de vida $^{15,16}$.

Tanto para los IECA como para los ARAII, se debe considerar el riesgo potencial en adolescentes con posibilidad de embarazarse, ya que pueden ocasionar hipotensión, bajo peso al nacer y descenso de la perfusión renal en el feto.

\section{Inhibidores de aldosterona sintetasa}

Los estudios que avalan su uso en manejo de hiperaldosteronismo primario. Además, publicaciones recientes en adultos, muestran una excelente respuesta a espironolactona como antihipertensivo de tercera línea en casos de HTA resistente a tratamiento con IECA, hidroclorotiazida y amlodipino ${ }^{17,18}$.

Es importante destacar que no está recomendado utilizar drogas que bloqueen el eje RAA en distintos puntos, por riesgo de: hiperkalemia, disfunción renal e hipotensión, por lo tanto, terapia dual podría ser utilizada en forma cautelosa en pacientes con proteinuria severa, bajo estricto monitoreo de función renal y kalemia.

\section{Vasodilatador periférico}

Hidralazina: Vasodilatador arteriolar se utiliza en hipertensión moderada a severa. En Chile, sólo disponible vía oral. Debe usarse con precaución debido a sus efectos secundarios, especialmente tipo lupus like.

Doxazocina: Bloqueador alfa adrenérgico que puede utilizarse en hipertensión leve a moderada. Especialmente útil al realizar estudio endocrinológico de HTA, ya que prácticamente no interfiere en los resultados.

Finalmente, la droga por elegir dependerá de la etiología de la HTA y del daño de órgano blanco ${ }^{3,6}$ (figura 1 ).

Para la dosificación y efectos adversos más frecuentes de los fármacos antihipertensivos (tabla 2).

\section{Esquemas hipotensores sugeridos según etiología de Hipertensión Arterial}

HTA asociada a obesidad: Desde el punto de vista terapéutico, los pilares del tratamiento son las modificaciones del estilo de vida y la reducción del peso ${ }^{2,19}$. En aquellos pacientes que sea necesario manejo farmacológico, los ensayos clínicos indican que todos los fármacos antihipertensivos poseen una eficacia similar en la reducción de la PA sistémica y daño de órgano relacionado con HTA en pacientes hipertensos obesos ${ }^{20}$. Sin embargo, algunos agentes antihipertensivos como beta bloqueadores o diuréticos tiazídicos, pueden tener efectos secundarios metabólicos y hemodinámicos no deseados como: reducción de la sensibilidad a la insulina, aumento de TG y LDL. Los bloqueadores de los canales de calcio son metabólicamente neutros ${ }^{21}$. Como se comentó, el SRAA en el tejido adiposo ha sido implicado en el desarrollo de HTA y la retención de sodio juega un papel central. Por lo tanto, tratamiento con un IECA, ARAII o ACC debe ser considerado como el tratamiento farmacológico antihipertensivo de primera línea en la hipertensión por obesidad ${ }^{19,20}$. Para evitar la retención de sodio y agua, pudiera adicionar un diurético tiazídico, pero siempre en combinación con el tratamiento ya descrito. En adolescentes mujeres, en edad fértil, es importante advertir potencial teratogénico de IECA y ARAII.

HTA asociada a corticoides: Presenta HTA por retención de sodio y agua, por lo cual se recomienda uso de diuréticos como hidroclorotiazida.

HTA asociada a DM: En estos pacientes, la HTA es un factor de riesgo de progresión de enfermedad y aparición de ERC, por lo cual se sugiere control estricto de PA. Se inicia terapia farmacológica, precozmente. Se prefiere IECA, ARAII o ACC. Incluso se recomienda inicio de terapia con perdida mantenida de variación circadiana de PA, ya que esta precede aparición de proteinuria. Se debe evitar el uso de diuréticos como tiazidas que pueden favorecer la hiperglicemia y de beta bloqueadores, que pueden enmascarar síntomas de hipoglicemia.

HTA en atletas: Se recomienda IECA, ARAII o ACC. Beta bloqueadores disminuyen resistencia física a ejercicio y diuréticos favorecen deshidratación.

HTA en ERC: Estudios han demostrados que la HTA está presente en $20-80 \%$ de los pacientes con ERC. Además, es conocido que el rango de PA afecta la progresión ERC, así pacientes con ERC y PAS > 120 $\mathrm{mmHg}$, tienen una velocidad de progresión mayor que pacientes con ERC normotensos. El deterioro es mayor aún frente a la ausencia de DIP nocturno.

En estudio ESCAPE, demostró que el control de PA bajo p50 en control $24 \mathrm{~h}$ (en pacientes con proteinuria moderada) disminuye la velocidad de progresión de ERC y aumenta sobrevida renal al cabo de 5 años ${ }^{22,23}$.

En estos pacientes, se sugiere iniciar tratamiento con IECA o ARA II. Si es necesario se puede adicionar otro hipotensor.

\section{Tratamiento de crisis hipertensiva}

\section{Emergencia hipertensiva}

La elección del medicamento debe hacerse en base a la etiología, tiempo de acción, vida media corta, seguridad y eficacia del medicamento.

Preferir medicamentos parenterales por ser mejor titulables, pues la PA no debe bajar bruscamente. Se recomienda $25-30 \%$ de baja en 6-8 h y luego disminuir gradualmente en 24-48 h hasta normotensión ${ }^{24}$. 


\section{Tabla 2. Fármacos hipotensores}

\begin{tabular}{|c|c|c|c|c|c|c|}
\hline Fármacos & Edad & Dosis inicial & Dosis máx. & $\mathrm{Fd}$ & Contraindicación & Efectos $2^{\circ}$ \\
\hline IECA & & & & & $\begin{array}{l}\text { Embarazo, } \\
\text { angioedema }\end{array}$ & $\begin{array}{l}\text { Cefalea, decaimiento, } \\
\text { hiperkalemia, IRA, daño fetal, } \\
\text { angioedema, tos }\end{array}$ \\
\hline Enalapril & $>1$ mes & $\begin{array}{l}0,08 \mathrm{mg} / \mathrm{Kg} / \mathrm{d} \\
\text { máx. } 5 \mathrm{mg} / \mathrm{d}\end{array}$ & $\begin{array}{l}0,6 \mathrm{mg} / \mathrm{Kg} / \mathrm{d} \max \\
40 \mathrm{mg} / \mathrm{d}\end{array}$ & $1-2$ & & \\
\hline \multirow[t]{2}{*}{ Captopril } & Lactante & $0,05 \mathrm{mg} / \mathrm{kg} / \mathrm{d}$ & $6 \mathrm{mg} / \mathrm{kg} \mathrm{d}$ & $1-4$ & & \\
\hline & Niño & $0,5 \mathrm{mg} / \mathrm{kg} / \mathrm{d}$ & & 3 & & \\
\hline ARA 2 & & & & & $\begin{array}{l}\text { Embarazo } \\
\text { angioedema }\end{array}$ & $\begin{array}{l}\text { Cefalea, decaimiento, } \\
\text { hiperkalemia, IRA, daño fetal }\end{array}$ \\
\hline Losartan & Niño & $\begin{array}{l}0,7 \mathrm{mg} / \mathrm{kg} / \mathrm{d} \\
\text { máx. } 50 \mathrm{mg}\end{array}$ & $\begin{array}{l}1,4 \mathrm{mg} / \mathrm{kg} / \mathrm{d} \text { máx. } \\
100 \mathrm{mg}\end{array}$ & $1-2$ & & \\
\hline Valsartan & Niño & $\begin{array}{l}1,3 \mathrm{mg} / \mathrm{kg} / \mathrm{d} \\
\text { máx. } 40 \mathrm{mg} / \mathrm{d}\end{array}$ & $\begin{array}{l}2,7 \mathrm{mg} / \mathrm{kg} / \mathrm{d} \text { máx. } \\
160 \mathrm{mg} / \mathrm{d}\end{array}$ & 1 & & \\
\hline$A C C$ & & & & & $\begin{array}{l}\text { Hipersensibilidad } \\
\text { insuficiencia cardiaca } \\
\text { congestiva }\end{array}$ & $\begin{array}{l}\text { Rubor facial, Decaimiento, } \\
\text { edema periférico, } \\
\text { angioedema }\end{array}$ \\
\hline Nifedipino AP & Niño & $0,2-0,5 \mathrm{mg} / \mathrm{Kg} / \mathrm{d}$ & $\begin{array}{l}3 \mathrm{mg} / \mathrm{kg} / \mathrm{d} \\
\text { máx. } 120 \mathrm{mg} / \mathrm{d}\end{array}$ & $1-2$ & & \\
\hline \multirow[t]{2}{*}{ Amlodipino } & $1-5 a$ & $\begin{array}{l}0,05- \\
0,1 \mathrm{mg} / \mathrm{kg} / \mathrm{d}\end{array}$ & $\begin{array}{l}0,6 \mathrm{mg} / \mathrm{kg} \mathrm{d} \\
\text { máx. } 5 \mathrm{mg} / \mathrm{d}\end{array}$ & 1 & & \\
\hline & $>6 a$ & $2,5 \mathrm{mg} \mathrm{d}$ & $10 \mathrm{mg} / \mathrm{d}$ & 1 & & \\
\hline $\begin{array}{l}\text { Diuréticos } \\
\text { tiazídicos }\end{array}$ & & & & & Anuria & $\begin{array}{l}\text { Decaimiento, hipokalemia, } \\
\text { arritmias, ictericia colestásica, } \\
\text { debut DM, pancreatitis }\end{array}$ \\
\hline Hidroclorotiazida & Niño & $1 \mathrm{mg} / \mathrm{kg} / \mathrm{d}$ & $\begin{array}{l}2 \mathrm{mg} / \mathrm{kg} / \mathrm{d} \\
\text { máx. } 37,5 \mathrm{mg} / \mathrm{d}\end{array}$ & $1-2$ & & \\
\hline Diuréticos de asa & & & & & Anuria & $\begin{array}{l}\text { Mareos, cansancio, alteraciones } \\
\text { hidroelectrolíticas }\end{array}$ \\
\hline Furosemida & & $0,5-2 \mathrm{mg} / \mathrm{kg} / \mathrm{d}$ & $6 \mathrm{mg} / \mathrm{kg} / \mathrm{d}$ & $1-2$ & & \\
\hline B.R.A. & & & & & $\begin{array}{l}\text { Insuficiencia renal } \\
\text { crónica }\end{array}$ & $\begin{array}{l}\text { Ginecomastia } \\
\text { hiperkalemia }\end{array}$ \\
\hline Espironolactona & & $1 \mathrm{mg} / \mathrm{kg} / \mathrm{d}$ & $\begin{array}{l}3,3 \mathrm{mg} / \mathrm{kg} / \mathrm{d} \\
\text { máx. } 100 \mathrm{mg} / \mathrm{d}\end{array}$ & $1-2$ & & \\
\hline Beta B. & & & & & Asma bronquial & Decaimiento, mareos \\
\hline Atenolol & & $0,5-1 \mathrm{mg} / \mathrm{kg} / \mathrm{d}$ & $\begin{array}{l}2 \mathrm{mg} / \mathrm{kg} / \mathrm{d} \text { max. } \\
100 \mathrm{mg} / \mathrm{d}\end{array}$ & 1 & & \\
\hline Propranolol & & $1 \mathrm{mg} / \mathrm{kg} / \mathrm{d}$ & $\begin{array}{l}1-8 \mathrm{mg} / \mathrm{kg} / \mathrm{d} \text { (máx. } \\
8 \mathrm{mg} / \mathrm{d})\end{array}$ & $2-4$ & & \\
\hline \multicolumn{7}{|l|}{ A.A. C } \\
\hline Clonidina & & $5-20 \mathrm{mcg} / \mathrm{kg} / \mathrm{d}$ & $\begin{array}{l}25 \mathrm{mcg} / \mathrm{kg} / \mathrm{d} \\
\text { (hasta 0,9 mg/d) }\end{array}$ & $3-4$ & $\begin{array}{l}\text { Hipersensibilidad, } \\
\text { bradiarritmia grave }\end{array}$ & $\begin{array}{l}\text { Depresión trastorno del } \\
\text { sueño, mareo, sedación, } \\
\text { cefalea }\end{array}$ \\
\hline \multicolumn{7}{|l|}{ V.D } \\
\hline Hidralazina & & $0,25 \mathrm{mg} / \mathrm{kg} / \mathrm{d}$ & $\begin{array}{l}7,5 \mathrm{mg} / \mathrm{kg} / \mathrm{d} \text { máx. } \\
200 \mathrm{mg} / \mathrm{d}\end{array}$ & $3-4$ & $\begin{array}{l}\text { Hipersensibilidad, } \\
\text { Lupus }\end{array}$ & Lupus like \\
\hline Minoxidil & & $0,1-0,2 \mathrm{mg} / \mathrm{kg} / \mathrm{d}$ & $\begin{array}{l}1 \mathrm{mg} / \mathrm{kg} / \mathrm{d} \text { máx. } 50 \\
\mathrm{mg} / \mathrm{d}\end{array}$ & $2-3$ & Insuficiencia cardiaca & $\begin{array}{l}\text { Prurito, irritación, } \\
\text { hipertricosis, edema }\end{array}$ \\
\hline
\end{tabular}

d: día, Fd: frecuencia día (número de veces/día), a: años, ACC: antagonista canal calcio, AP: acción prolongada, IECA: inhibidor enzima convertidora de angiotensina, ARA: antagonista receptor angiotensina, BRA: bloqueador receptor aldosterona, BetaB: betabloqueador, AAC: alfa agonista central. VD: vasodilatadores. 
Nicardipino es el medicamento de elección, pero no está disponible en Chile. Es un bloqueador de canal de calcio dihidropiridínico, que disminuye la resistencia vascular periférica, sin efecto negativo en inotropismo. Se puede usar en broncoespasmo, falla hepática y renal. Vida media 10-15 min, su efecto se inicia en $15 \mathrm{~min}$. Efectos secundarios: taquicardia y flashing. No hay riesgo de toxicidad por tiocianato.

Labetalol: es un alfa y beta bloqueador, que disminuye la RVP. Vida media de 3-5 h. No se puede usar en broncoespasmo ni en insuficiencia cardiaca congestiva por efecto inótropo negativo. Precaución en pacientes diabéticos, además puede empeorar hiperkalemia. Se puede utilizar en bolo y en infusión continua ${ }^{24}$.

Hidralazina: se usa principalmente en neonatos y embarazadas, pero no está disponible en Chile en su presentación parenteral. Es un potente vasodilatador arterial, vida media de 2-4 h. Puede utilizarse inicialmente intramuscular. Efectos secundarios: taquicardia y retención de sodio $^{24}$.

Nitroprusiato de sodio: tiene un rápido tiempo de acción. Vasodilatador venoso y arterial que disminuye la pre y post carga, siendo beneficioso en casos de insuficiencia cardiaca congestiva inducida por HTA. Tras 24-48 hrs de uso, puede generar toxicidad por tiocianato, especialmente en presencia de falla renal y hepática, además puede aumentar la presión intracraneana ${ }^{24}$.

Una vez controlada la HTA y con paciente consciente, $24-48 \mathrm{~h}$ se puede cambiar a medicamentos orales de vida media más larga, mientras la velocidad de infusión de los antihipertensivos parenterales se va disminuyendo lentamente. El uso prolongado de antihipertensivos parenterales lleva a retención de agua

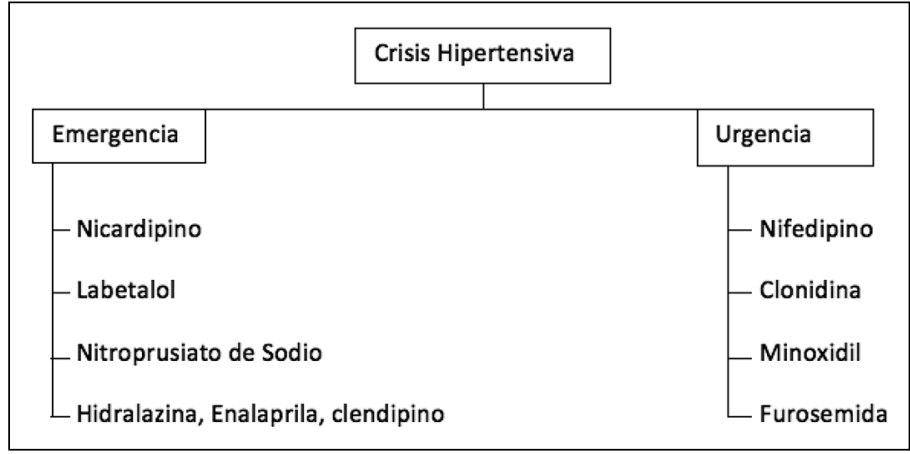

Figura 2. Fármacos sugeridos para el manejo de la crisis hipertensiva.

y sodio.

\section{Urgencia hipertensiva}

Si el paciente es asintomático, hay tiempo para reducción gradual de PA con medicamentos orales. Se puede utilizar nifedipino en la menor dosis efectiva recomendada. En niños mayores se puede utilizar clonidina o minoxidil.

Clonidina: es un alfa 2 agonista central que disminuye el estímulo adrenérgico cerebral. Inicio de acción en 15-30 min, se puede usar en falla renal. En exceso produce sedación y boca seca.

En casos de HTA renovascular se recomienda el uso de IECA o ARAII con seguimiento cuidadoso. No usar en monorreno o estenosis renal bilateral ${ }^{24}$ (figura 2).

\section{Conflicto de Intereses}

Los autores declaran no tener conflicto de intereses.

\section{Referencias}

1. Flynn JT, Kaelber DC, Baker-Smith $\mathrm{CM}$, et al. Clinical Practice Guideline for Screening and Management of High Blood Pressure in Children and Adolescents. Pediatrics 2017;140 (3): e20171904.

2. Urbina E, Agabiti-Rosei E, Cruickshank J, et al. European Society of Hypertension guidelines for the management of high blood pressure in children and adolescents. J Hipertens. 2016;34:1887920.

3. Lurbe E, Cifkova R, Cruickshank JK, et al. Manejo de hipertensión arterial en niños y adolescentes: recomendaciones de la Sociedad Europea de Hipertension. An Pediatr (Barc) 2010;73(1):51.e1-51.e28.
4. Lurbe E, Álvarez J, Redon J. Diagnosis and Treatment of Hipertension in Children. Curr Hypertens Rep 2010;12:480-6.

5. Anyaegbu E, Dharnidharka V. Hipertension in the Teenager. Pediatr Clin N Am 2014;61:131-51.

6. Flynn JT, Daniels SR, Hayman LL, et al. American Heart Association Atherosclerosis, Hypertension and Obesity in Youth Committee of the Council on Cardiovascular Disease in the Young. Update: ambulatory blood pressure monitoring in children and adolescents: a scientific statement from the American Heart Association. Hypertension 2014;63(5):111635. https://www.ncbi.nlm.nih.gov/ pubmed/24591341.

7. Batisky DL, Sorof JM, Sugg J, et al.
Toprol-XL Pediatric Hypertension Investigators. Efficacy and safety of extended release metoprolol succinate in hypertensive children 6 to 16 years of age: a clinical trial experience. J Pediatr 2007;150:134-9.

8. Cice G, Ferrara L, D'Andrea A, et al. Carvedilol Increases Two-Year Survival in Dialysis Patients With Dilated Cardiomyopathy. A Prospective, PlaceboControlled Trial. J Am Coll Cardiol 2003; 41:1438-44.

9. Flynn JT, Warnik SJ. Isradipine treatment of hypertension in children: a singlecenter experience. Pediatr Nephrol 2002;17:748-53.

10. Flynn JT, Newburger JW, Daniels SR, Sanders SP, Portman RJ, Hogg RJ. A randomized, placebo-controlled trial of 
amlodipine in children with hypertension. J Pediatr 2004;145: 353-9.

11. Flynn JT, Nahata MC, Mahan Jr JD, Portman RJ, PATH-2 Investigators. Population pharmacokinetics of amlodipine in hypertensive children and adolescents. J Clin Pharmacol 2006;46:905-16.

12. Wells T, Frame V, Soffer B, et al. Enalapril Pediatric Hypertension Study Group. A double-blind, placebo-controlled, dose-response study of the effectiveness and safety of enalapril for children with hypertension. J Clin Pharmacol 2002;42: 870-80.

13. Li JS, Berezny K, Kilaru R, et al. Is the extrapolated adult dose of fosinopril safe and effective in treating hypertensive children? Hypertension 2004;44:289-93.

14. Soffer B, Zhang Z, Miller K, Vogt BA, Shahinfar S. A doubleblind, placebocontrolled, dose-response study of the effectiveness and safety of lisinopril for children with hypertension. Am J
Hypertens 2003;16:795-800.

15. Croxtall JD. Valsartan: in children and adolescents with hypertension. Paediatr Drugs 2012;14(3):201-7.

16. Schaefer F, Coppo R, Bagga A at al. Efficacy and safety of valsartan in hypertensive children 6 months to 5 years of age. J Hypertens 2013;31(5):993-1000.

17. Karns AD, Bral JM, Hartman D et al. Study of aldosterone synthase inhibition as an add-on therapy in resistant hypertension. J Clin Hypertension (Greenwich) 2013;15(3):186-92.

18. Bobrie G, Frank M, Azizi M et al. Sequential nephron blockade versus sequential renin-angiotensin system blockade in resistant hypertension: a prospective, randomized, open blinded end point study. J Hypertens 2012; 30(8):1656-64. https://www.ncbi.nlm.nih. gov/pubmed/22728905.

19. Kotsis V, Stabouli S, Papakatsika S, Rizos Z, Parati G. Mechanisms of obesityinduced hypertension. Hypertension
Research 2010;33:386-393.

20. Wenzel U, Benndorf R, Lange S. Treatment of Arterial Hypertension in Obese Patients. Semin Nephrol 2013;33:66-74.

21. Landsberg L, Aronne L, Beilin L, et al. Obesity-Related Hypertension: Pathogenesis, Cardiovascular Risk, and Treatment. J Clin Hypertens 2013;15:1433.

22. Wühl E, Mehls O, Schaefer F, ESCAPE Trial Group. Antihypertensive and antiproteinuric efficacy of ramipril in children with chronic renal failure. Kidney Int 2004;66:768-76.

23. Wühl E, Mehls O, Schaefer F, ESCAPE trial group. Long-term dissociation of antiproteinuric and antihypertensive efficacy of ACE inhibition in children with chronic renal failure. Pediatr Nephrol 2006;21:1505.

24. Jayanthi C, Zilleruelo G. Hipertensive crisis in Children. Pediatr Nephrol 2012; 27:741-51. 